\title{
Formation and Evolution of E3 centers in Hydrothermally grown Zinc Oxide
}

\author{
A. Hupfer, C. Bhoodoo, L. Vines, and B. G. Svensson \\ University of Oslo, Physics Department/Center for Materials science \\ and Nanotechnology, P.O. Box 1048 Blindern, Oslo N-0316, Norway
}

(Dated: December 2, 2016)

\begin{abstract}
The formation and evolution of the prominent and so-called E3 center in $\mathrm{ZnO}$ has been studied by in-situ deep level transient spectroscopy measurements after on-line implantation of hydrogen $(\mathrm{H})$ and deuterium (D) ions at sample temperatures of $\sim 158 \mathrm{~K}$ and $\sim 285 \mathrm{~K}$. The formation of E3 is shown to involve migration and subsequent trapping of interstitial hydrogen $\left(\mathrm{H}_{i}\right)$, or deuterium, and starts to occur already below $200 \mathrm{~K}$. The concentration of implantation-induced E3 centers is rather unstable and decreases gradually at temperatures around $300 \mathrm{~K}$ by an annealing process obeying first-order kinetics. The process exhibits an activation energy of $\sim 0.85 \mathrm{eV}$ and involves presumably trapping of migrating $\mathrm{H}_{i}$ 's leading to passivation of the E3 centers. Further, the concentration of E3 centers is found to decrease rapidly during annealing in forming gas ambient at room temperature and then to recover gradually during subsequent annealing in vacuum. The E3 center is tentatively attributed to a complex between a $\mathrm{Zn}$ vacancy and three hydrogen atoms $\left(\mathrm{H}_{3} V_{\mathrm{Zn}}\right)$, supported by recent theoretical results showing that a $V_{\mathrm{Zn}}$ in $\mathrm{ZnO}$ can accommodate up to five hydrogen atoms in stable configurations [Hupfer et al. to be published (2016)].
\end{abstract}

\section{INTRODUCTION}

Hydrogen in semiconducting oxides can play many roles. In interstitial form it eften exhibits an amphoteric behavior [1. Substitutional $\mathrm{H}$ acts as a donor [20], with the ability to compensate acceptors, and it is a strong promotor of the commonly observed n-type conductivity of $\mathrm{ZnO}$. Given typical growth conditions, hydrogen is almost always present as a residual impurity in $\mathrm{ZnO}$ irrespective of the growth technique used. $\mathrm{H}$ gives also rise to deep level defects, and it was recently shown to be involved in the prominent defect level at $\sim E_{c}-0.3 \mathrm{eV}\left(E_{c}\right.$ denotes the conduction band edge) which is observed in most $\mathrm{ZnO}$ materials [11. The $E_{c}-0.3 \mathrm{eV}$ level was first reported by Simpson and Cordaro [2] and later characterized in more detail and labeled E3 by Auret et al. [3, 4, who showed that E3 is not influenced by $\mathrm{MeV}$ electron or proton irradiation. Furthermore, the concentration of E3 is also not affected by implantation of self-ions, i.e., $\mathrm{O}$ and $\mathrm{Zn} \mathrm{[5,} \mathrm{6],} \mathrm{while} \mathrm{seemingly} \mathrm{contradicting} \mathrm{results}$ are reported on the dependence on the annealing ambient [7 9]. Quemener et al. 8] found variations in the concentration of E3 by about one order of magnitude between nominally identical hydrothermal samples, implying that the growth conditions have a crucial impact on the E3 formation. However, post-growth treatment of these samples in Zn-rich and O-rich ambient under thermodynamic equilibrium condition did not affect the E3 concentration, indicating an impurity-related defect rather than an intrinsic one [10. Indeed, Hupfer et al. [11. presented recently unambiguous evidence for the involvement of $\mathrm{H}$ in the $\mathrm{E} 3$ center by an enhancement of its concentration of more than one order of magnitude after $\mathrm{H}$ implantation at room temperature (RT) using doses in the $10^{12} \mathrm{~cm}^{-2}$ range.

The atomic structure of E3 is not known as well as the mechanisms controlling its formation and annealing. In the present study, we show that E3 starts to form already at temperatures below $200 \mathrm{~K}$ using in-situ deep level transient spectroscopy (DLTS) measurements after hydrogen (H) / deuterium (D) implantation at cryogenic temperatures. Contrary to that of the free charge carriers (electrons), the formation of $\mathrm{E} 3$ involves migration and trapping of interstitial $\mathrm{H} / \mathrm{D}\left(\mathrm{H}_{i} / \mathrm{D}_{i}\right)$. The excess concentration of introduced E3 centers is rather unstable and disappears at RT via a first-order kinetics process, possibly caused by trapping of migrating $\mathrm{H}_{i}$ 's $\left(\mathrm{D}_{i}\right.$ 's). Finally, E3 is found to decrease in concentration after annealing in forming gas ambient at RT and then to gradually recover during subsequent annealing in vacuum $\left(\lesssim 1 \times 10^{-7} \mathrm{mBar}\right)$; this implies H-passivation of the donor-like E3 center and in accordance with recent theoretical predictions for complexes of zinc vacancies $\left(V_{\mathrm{Zn}}\right.$ 's) decorated with H's [Hupfer et al. to be published (2016)], E3 is tentatively attributed to a $V_{\mathrm{Zn}_{3}} \mathrm{H}_{3}$ complex.

\section{EXPERIMENTAL}

Wafers of hydrothermally grown n-type $\mathrm{ZnO}$ (HT$\mathrm{ZnO}$ ) purchased from Tokyo Denpa were cut into $5 \times 5 \mathrm{~mm}^{2}$ sized samples. The samples were cleaned in acetone and ethanol. After a $40 \mathrm{~s}$ treatment in boiling $\mathrm{H}_{2} \mathrm{O}_{2}(31 \%)$, 100nm thick Pd Schottky contacts were deposited on the Zn-polar face using electron-beam evaporation. The Schottky contacts displayed a rectification of more than two orders of magnitude between forward and reverse bias $(2 \mathrm{~V}$ and $-2 \mathrm{~V})$. In all measurements we carefully monitored conductance versus bias voltage which did not raise any concern in respect to leakage current from a possible deterioration of the diode. The samples were then implanted either with $325 \mathrm{keV}$ protons $\left(\mathrm{H}^{+}\right)$or deuterium ions $\left(\mathrm{D}^{+}\right)$, with a projected range of $\approx 2.0 \mu \mathrm{m}$, as estimated by Monte Carlo simulations using the SRIM code [12. The doses used were in the range of $5 \times 10^{10}$ to $1.4 \times 10^{12} \mathrm{~cm}^{-2}$ and the implatations 
were performed in an online setup for in-situ sample characterization by capacitance-voltage (CV) and deep level transient spectroscopy (DLTS) measurements at our laboratory. The sample temperature during implantation was either $\sim 158 \mathrm{~K}$ or $\sim 285 \mathrm{~K}$ and the DLTS measurements were then carried out while scanning the sample temperature from 100 to $250 \mathrm{~K}$ using a refined version of the system described in 13 . A reverse bias of $-7 \mathrm{~V}$ was applied with a filling pulse of $7 \mathrm{~V}$ and $50 \mathrm{~ms}$ duration. The bulk net carrier concentration was determined through $\mathrm{CV}$ measurements ( $1 \mathrm{MHz}$ probing frequency) in the scanned temperature range. For E3 concentration versus depth profiling a single rate window was applied, and the temperature was held constant within $\pm 0.5 \mathrm{~K}$ at the maximum of the studied E3 peak, e.g., $158 \mathrm{~K}$ for a rate window of $(640 \mathrm{~ms})^{-1}$. The steady-state bias voltage was kept constant while gradually increasing the amplitude of the filling pulse, and the depth profile was subsequently extracted from the dependence of the DLTS signal on the pulse amplitude.

\section{RESULTS AND DISCUSSION}

\section{A. Formation of E3 during ion implantation}

Figure 1 a) shows the net charge carrier concentration $\left(N_{d}\right)$ versus depth extracted from CV measurements before and after implantation with $325 \mathrm{keV}$ deuterium ions (dose $1 \times 10^{12} \mathrm{~cm}^{-2}$ ) at a sample temperature of $158 \mathrm{~K}$. Prior to the implantation, the samples exhibited an almost uniform $N_{d}$ profile of $\approx 8 \times 10^{14} \mathrm{~cm}^{-3}$. The calculated profile in Fig. 1 a) was obtained using the SRIM code 12 (dashed lines, shown at $1 / 3$ rd of scale). After the $158 \mathrm{~K}$ implantation (Fig 1 (a)), an immediate increase in $N_{d}$ is observed which generally follows the implantation profile. Because of this increase in $N_{d}$ and the maximum applicable reverse bias voltage remaining the same, the probed region becomes more shallow with dose and the profiles cannot be monitored over the whole peak depth range. The increase in $N_{d}$ is, indeed, attributed to the implanted D atoms, which like hydrogen can act as a shallow donor but also exhibit a strong reactivity with impurities/traps and efficiently passivate acceptorlike defects [14. At $158 \mathrm{~K}$, the diffusivity of $\mathrm{D}$ is very low [15] and only short range interaction is anticipated; thus, the increase in $N_{d}$ is primarily ascribed to the implanted $\mathrm{D}$ atoms adopting an interstitial configuration acting as shallow donors. A comparison with the calculated profile in Fig. 1 (a) indicates that the interstitial fraction of the implanted D atoms is on the order of $\sim 30 \%$. Moreover, the increase in $N_{d}$ remains stable when the temperature is raised from $158 \mathrm{~K}$ to $290 \mathrm{~K}$. Figure $1 \mathrm{~b}$ ) shows the E3 concentration versus depth profiles for the corresponding conditions as in Fig. 1 (a). In contrast to $N_{d}$, the increase in E3 does not occur immediately after the $158 \mathrm{~K}$ implantation but starts at higher temperatures $(\gtrsim 170 \mathrm{~K})$ and continues at least up to $290 \mathrm{~K}$. This implies a ther-
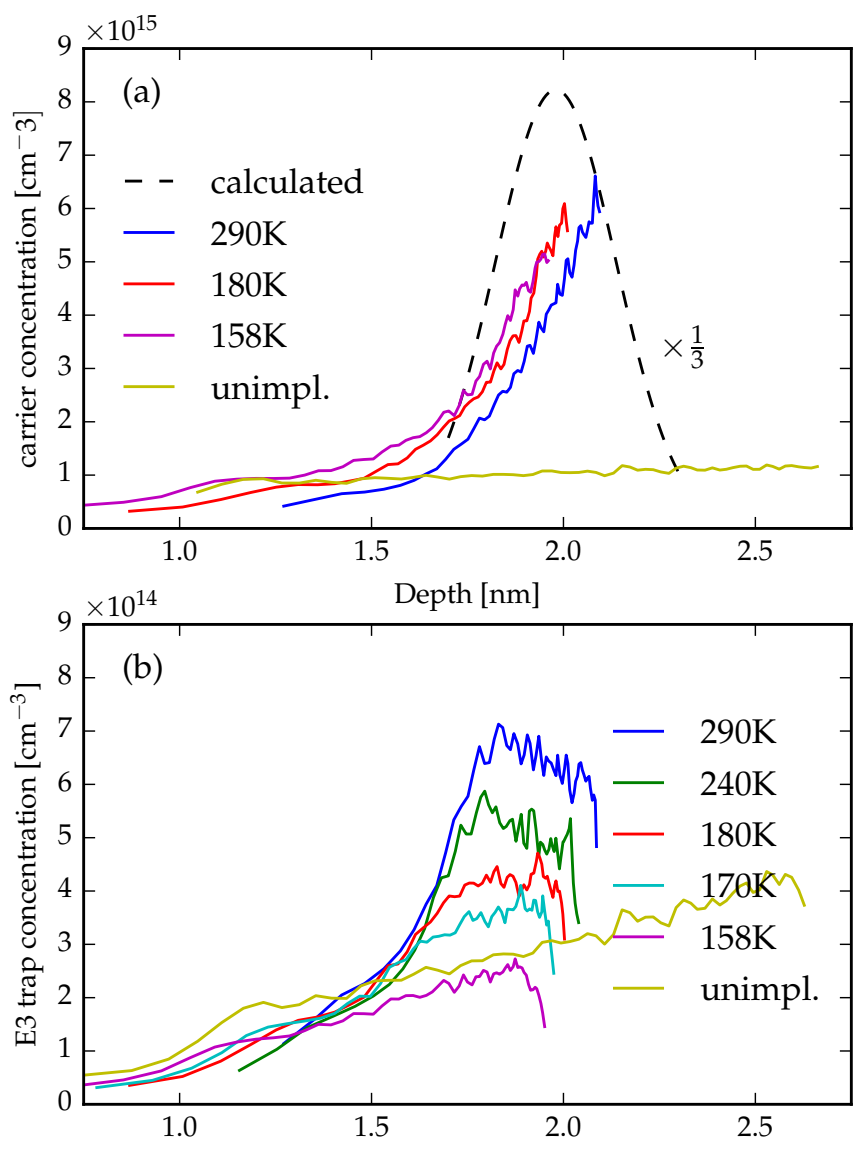

Figure 1: Influence of isochronal annealing temperature following deuterium implantation $\left(1 \times 10^{12} \mathrm{~cm}^{-2}\right)$ on the charge concentration a) and the E3 trap concentration b). The calculated profile in a) was obtained using the SRIM-code 12 and is shown at $1 / 3$ rd of its scale.

mally activated process with migration of $\mathrm{D}_{i}$ as a likely candidate.

To investigate the dependency of the E3 concentration on the implantation dose, two nominally identical samples have been implanted at $285 \mathrm{~K}$ with a series of successively increasing $\mathrm{H}$ and $\mathrm{D}$ doses. $N_{d}$ and E3 profiles were measured after each dose, and the results are depicted in Figs 2 (a) and (b) respectively. In the insets of Figs. 2 (a) and (b), the data for the peak concentration in the $\mathrm{H}$ and $\mathrm{D}$ implanted samples are compared. The shape of the $N_{d}$ and E3 profiles remain essentially the same as a function of the accumulated dose and the peak values increase linearly with both the $\mathrm{H}$ and $\mathrm{D}$ dose, except for an apparent saturation in the E3 concentration at the two highest $\mathrm{H}$ doses used. We have shown previously [? ] that implantation of He in nominally identical samples with similar projected ranges do not produce an increase in charge carrier concentration. Therefore any observed increase in charge carrier concentration can unambiguously be attributed to the implanted species rather then formation of intrinsic defects. The rate of increase for 

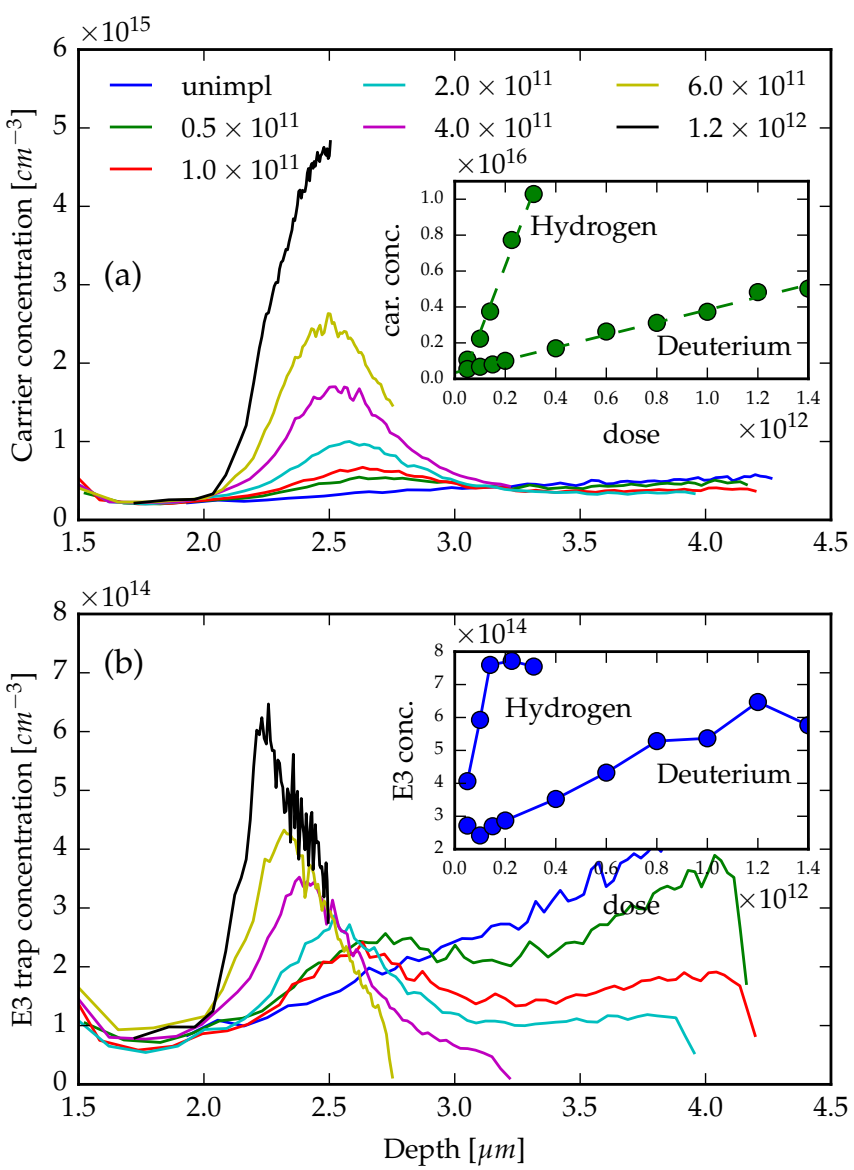

Figure 2: Implantation dose dependency of $N_{d}$ (a) and E3 concentration (b). The implantations were undertaken at $285 \mathrm{~K}$ sample temperature. The profiles shown are taken from the D-implanted samples, and the insets display the profile peak values versus ion dose.

both $N_{d}$ and E3 are, however a factor of $\sim 10$ higher in the H-implanted samples relative to the D-implanted ones. In fact, the increase of $N_{d}$ in the former samples amounts to more than $95 \%$ of the implanted $\mathrm{H}$ concentration, implying that almost all of the $\mathrm{H}$ atoms act as shallow donors with $\mathrm{H}_{i}$ as the prevailing configuration. For the D-implated samples, the corresponding apparent fraction is only $\sim 15 \%$ and actually lower than that after the $158 \mathrm{~K}$ implant $(\sim 30-35 \%$, Fig. 1 (a)). This low apparent fraction of $\mathrm{D}_{i}$ can be associated with the higher defect generation rate per $\mathrm{D}^{+}$ion relative to that for a $\mathrm{H}^{+}$ion, with a difference by a factor of $\sim 3$ in the implantation peak region, as estimated from SRIM simulations. Accordingly, the probability for trapping of migrating $\mathrm{D}_{i}$ 's by implantation-induced intrinsic defects leading to loss of the shallow $\mathrm{D}_{i}$ donors is higher than that for $\mathrm{H}_{i}$. At $158 \mathrm{~K}, \mathrm{D}_{i}$ is almost immobile and a large fraction remains in the interstitial configuration without reacting with the ion-induced intrinsic defects. Furthermore, $\mathrm{Zn}_{i}$, acting as a shallow double donor, is expected to become mobile around RT and interact with other de- fects, giving rise to a loss in the net carrier concentration 16. That is, the initial balance between the generated Frenkel pairs of $\mathrm{Zn}_{i}$ and $V_{\mathrm{Zn}}$ is no longer valid and hence, the actual fraction of $\mathrm{D}_{i}$ may be larger than that estimated from Fig. 2 (a), because of compensation by the deep $V_{\mathrm{Zn}}$ acceptors.

For E3, the absolute increase in concentration with ion dose is about a factor of 10 lower than that for $N_{d}$, c.f. both the $\mathrm{H}$ - and $\mathrm{D}$ - implanted samples in Fig. 2, The plateau occurring after a H-dose of $\sim 2 \times 10^{11} \mathrm{~cm}^{-2}$ is due to the increase in $N_{d}$ and the limit of the maximum applicable reverse bias voltage, preventing monitoring of the whole profile peak, similar to that for $N_{d}$ in Fig. 11. In the H-samples, E3 evolves almost instantaneously during the short duration $(\sim 1-2 \mathrm{~min})$ of implantation at $285 \mathrm{~K}$ before cooling down to the DLTS measurement temperature of $\sim 158 \mathrm{~K}$. However, in the D-samples the E3 evolution is considerably slower and durations on the order of $\sim 1 \mathrm{~h}$ at $285 \mathrm{~K}$ are required to reach the maximum concentration. That is, the E3profiles and values depicted in Fig. 2 for the D-samples do not represent the fully developed (saturated) concentration but the initial ones after $1-2$ min duration. The evolution of the E3-profiles with annealing time at $300 \mathrm{~K}$ is shown in Fig. 3 (b) for a D-sample implanted at $158 \mathrm{~K}$ to a dose of $10^{12} \mathrm{~cm}^{-2}$ and subsequently isochronally annealed (10 $\mathrm{min})$ at 170, 180, 240 and $290 \mathrm{~K}$, c.f. Fig. 1. The peak concentration increases from a starting value of $\sim 7 \times 10^{14}$ to $\sim 2.5 \times 10^{15} \mathrm{~cm}^{-3}$ after $\sim 2 \mathrm{~h}$ before a gradual decrease occurs. The difference in E3 generation rate becomes now only a factor of $\sim 3$ (and not $\sim 8$ ) between the $\mathrm{H}$ - and D-samples when considering the maximum (saturated) concentration values. Accordingly and as also corroborated by the data in Fig. 1(b), $\mathrm{D}_{i}$ (and $\mathrm{H}_{i}$ ) migration is a necessary condition for the formation of E3 centers, with the migration of $\mathrm{H}_{i}$ being faster then that of $\mathrm{D}_{i}$. Finally, the linear increase in the E3 concentration up to at least a few times $10^{15} \mathrm{~cm}^{-3}$ with ion dose implies that the formation process invokes trapping of the migrating $\mathrm{D}_{i}$ 's (and $\mathrm{H}_{i}$ 's) by an abundant defect/impurity. It is tempting to speculate that the trap involves one of the implantation-induced primary point defects, especially $V_{\mathrm{Zn}}$ as will be discussed in section IV.

\section{B. Annealing kinetics of the E3 center}

Figure 3 shows the evolution of the E3 and $N_{d}$ profiles during isothermal annealing at $300 \mathrm{~K}$ of a sample implanted with D at $158 \mathrm{~K}\left(1 \times 10^{12} \mathrm{~cm}^{-2}\right)$ and subjected to isochronal annealing up to $290 \mathrm{~K}$, c.f. Fig. 1. $N_{d}$ decreases continuously with the annealing time whilst E3 increases initially, before decreasing.

Figure 4 displays the profile peak concentrations of $N_{d}$ and E3 centers versus annealing time at $300 \mathrm{~K}$, extracted from their depth-distributions exemplified in Fig. 3. In the D-samples, $N_{d}$ exhibits an initial and rapid decrease during the first $\sim 30 \mathrm{~min}$ and for the E3 a corresponding 

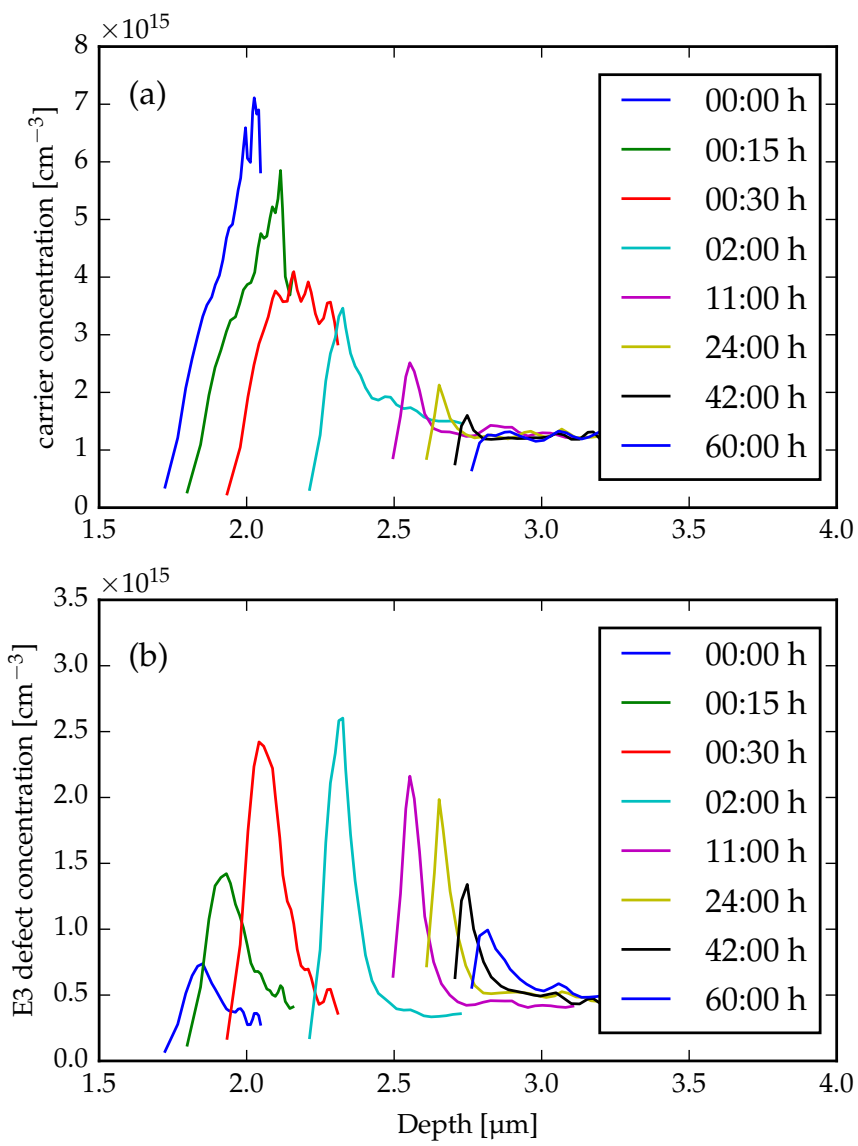

Figure 3: Influence of isothermal annealing time at $300 \mathrm{~K}$, following the isochronal annealing in Fig. 1 on $N_{d}$ a) and E3 concentration b). The profiles are displaced in depth for clarity.

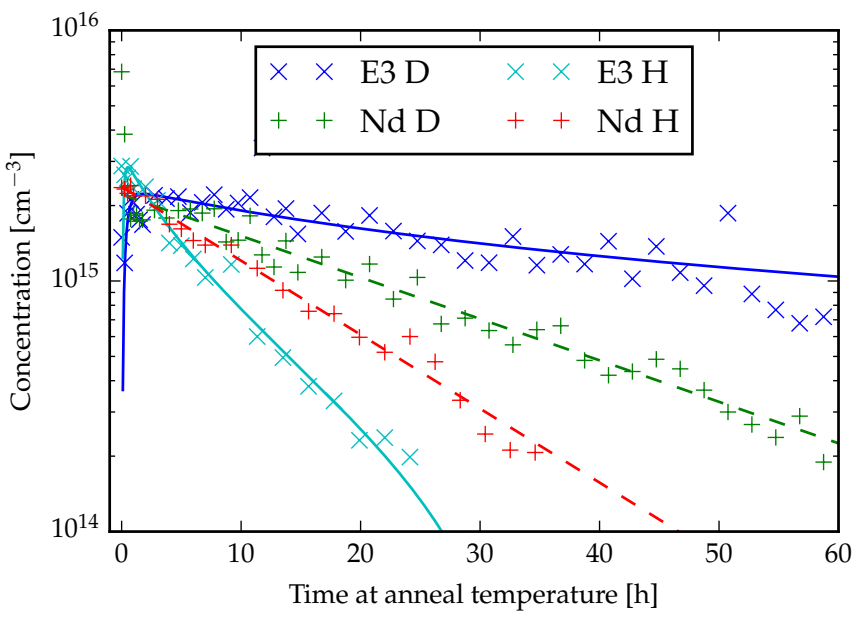

Figure 4: time dependence of the peak concentrations of excess E3 centers and excess charge carriers during isothermal annealing at $300 \mathrm{~K}$ after deuterium and hydrogen implantation $\left(1 \times 10^{12} \mathrm{~cm}^{-2}, 158 \mathrm{~K}\right)$. increase takes place. Subsequently, both $N_{d}$ and E3 show an exponential decay but with a larger rate constant for $N_{d}$. In the H-samples, a rapid initial decrease and increase of $N_{d}$ and E3, respectively, are not resolved before the exponential decay starts. This is probably because of an even more rapid process than in the D-samples and that the peaks of the profiles are not possible to access initially due to their high concentrations after the dose of $1 \times 10^{12} \mathrm{H} / \mathrm{cm}^{2}$. The decay rate of $N_{d}$ is about a factor of 1.4 higher in the H-samples compared to the D-samples and reflects presumably the difference in diffusivity between $\mathrm{H}_{i}$ and $\mathrm{D}_{i}$. For $\mathrm{E} 3$, the rate constants differ by a factor of $\sim 5.5$ in the two samples and may indicate an influence on the annealing process by the ratio between the concentrations of implanted species and generated defects. According to SRIM simulations [12] and assuming a survival factor of $2 \%$ during dynamic annealing [17, the ratio between implanted ions and generated $V_{\mathrm{Zn}}$ 's in the peak region becomes $\sim 18$ and $\sim 6$ for the $\mathrm{H}^{+}$and $\mathrm{D}^{+}$ions, respectively. Hence, a high ratio appears to promote the E3 loss rate and indicates that $\mathrm{H}_{i}$ and $\mathrm{D}_{i}$ play a role in the annealing process.

Figure 5 shows the Arrhenius plot of the rate constant values deduced for the loss of $\mathrm{E} 3$ in four samples implanted with $\mathrm{H}$ under identical conditions $\left(8 \times 10^{11} \mathrm{~cm}^{-2}\right.$, $285 \mathrm{~K}$ ) and then isothermally annealed at $290 \mathrm{~K}, 300 \mathrm{~K}$, $310 \mathrm{~K}$, and $315 \mathrm{~K}$, respectively. An activation energy of $\sim 0.87( \pm 0.05) \mathrm{eV}$ is obtained with a pre-exponential factor of $\sim 2 \times 10^{10} \mathrm{~s}^{-1}$. A first order kinetics process (exponential decay) for the loss of E3 can occur via either dissociation of the defect center or reaction with another defect/impurity having about one order of magnitude (or more) higher concentration than E3. In the first case, a pre-exponential factor between $10^{12}$ and $10^{13} \mathrm{~s}^{-1}$ is expected. Thus, despite a considerable uncertainty by up to one order of magnitude in the value extracted for the pre-factor, dissociation is excluded as a likely process for the loss of E3. Instead, a reaction with a species $\mathrm{X}$ of sufficient concentration and diffusivity is favored and applying the theory for diffusion-limited reactions [18, 19], one arrives at the condition $D_{x_{0}}[X] \simeq 10^{16} \mathrm{~cm}^{-1} \mathrm{~s}^{-1}$ in order to account for the pre-factor of $\sim 10^{10} \mathrm{~s}^{-1}\left(D_{x_{0}}\right.$ denotes the pre-exponential factor of the diffusivity of $X$ and $[X]$ is the concentration of $X$ ). Considering that the implanted $\mathrm{H}$ concentration in the peak region is $\sim 4 \times 10^{16} \mathrm{~cm}^{-3}$ for the studied samples, as estimated from SRIM simulations [12, $\mathrm{H}_{i}$ emerges as a viable candidate for $X$. Moreover, $\mathrm{H}_{i}$ is quite mobile in the range of annealing temperatures employed and the activation energy of $\sim 0.87 \mathrm{eV}$ would then represent the migration energy of $\mathrm{H}_{i}$ plus a contribution from a possible reaction barrier between $\mathrm{H}_{i}$ and E3. Here, it should be underlined that the migration process must be of short range $(<100 \mathrm{~nm})$ since secondary ion mass spectrometry (SIMS) measurements did not reveal any broadening of corresponding D concentration-versus-depth profiles after the E3 annealing (data not show); 30 Further evi- 


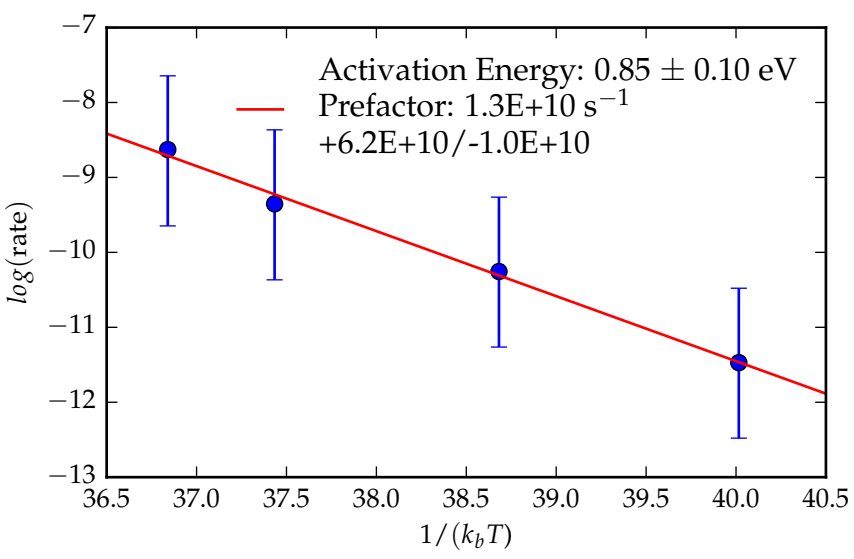

Figure 5: Arrhenius plot of the annealing rate constants for the peak concentration of E3 centers after hydrogen implantation. The rate constants were deduced from isothermal anneals performed at 290, 300, 310 and $315 \mathrm{~K}$.

dence for the involvement of $\mathrm{H}$ in the annealing process of E3 centers is given in the following section.

\section{Influence of annealing ambient on E3}

Figure 6 shows the depth-profiles of a) $N_{d}$ and b) the E3 concentration, without background subtraction after the final annealing stage in Fig. 4. This sample was then exposed to an atmosphere of forming-gas $\left(\mathrm{N}_{0.9} \mathrm{H}_{0.1}\right)$ at a pressure of $5 \mathrm{mBar}$ at $290 \mathrm{~K}$ for $15 \mathrm{~min}$. Thereafter, the sample was cooled down to $158 \mathrm{~K}$ and the ambient condition was restored to vacuum $\left(<1 \times 10^{-7} \mathrm{mBar}\right) . N_{d}$ and E3 were then monitored by CV and DLTS measurements at $158 \mathrm{~K}$, respectively, after sequential isothermal annealing at $300 \mathrm{~K}$ for durations up to $28 \mathrm{~h}$. The treatment in forming gas ambient leads to a substantial reduction of $N_{d}$ and almost complete vanishing of E3. However, during the subsequent $300 \mathrm{~K}$ annealing, both $N_{d}$ and E3 recover gradually and eventually, their concentrations approach the initial ones at $t=0$ in Fig. 4. Here, it should be underlined that the contribution from E3 to the recorded $N_{d}$ profile in Fig. 6 (a) is strong and the evolution of $N_{d}$ is to a large extent governed by that of E3.

The data in Fig. 6 comply strongly with the conclusion that the E3 centers do not break up (dissociate) during the post-implant annealing but are passivated by the excess of $\mathrm{H}_{i}$ (or $\mathrm{D}_{i}$ ) introduced. The forming gas anneal enhances this excess even further and also the centers present prior to the implantation become passivated. The recovery of E3 during the $300 \mathrm{~K}$ annealing after the forming-gas treatment implies that the stability of the passivated E3 centers is low and they dissociate by releasing $\mathrm{H}_{i}$ 's and/or $\mathrm{H}_{2}$ molecules. This is in contrast to the samples subjected to post-implant annealing only where the E3 centers remain absent and do not reappear
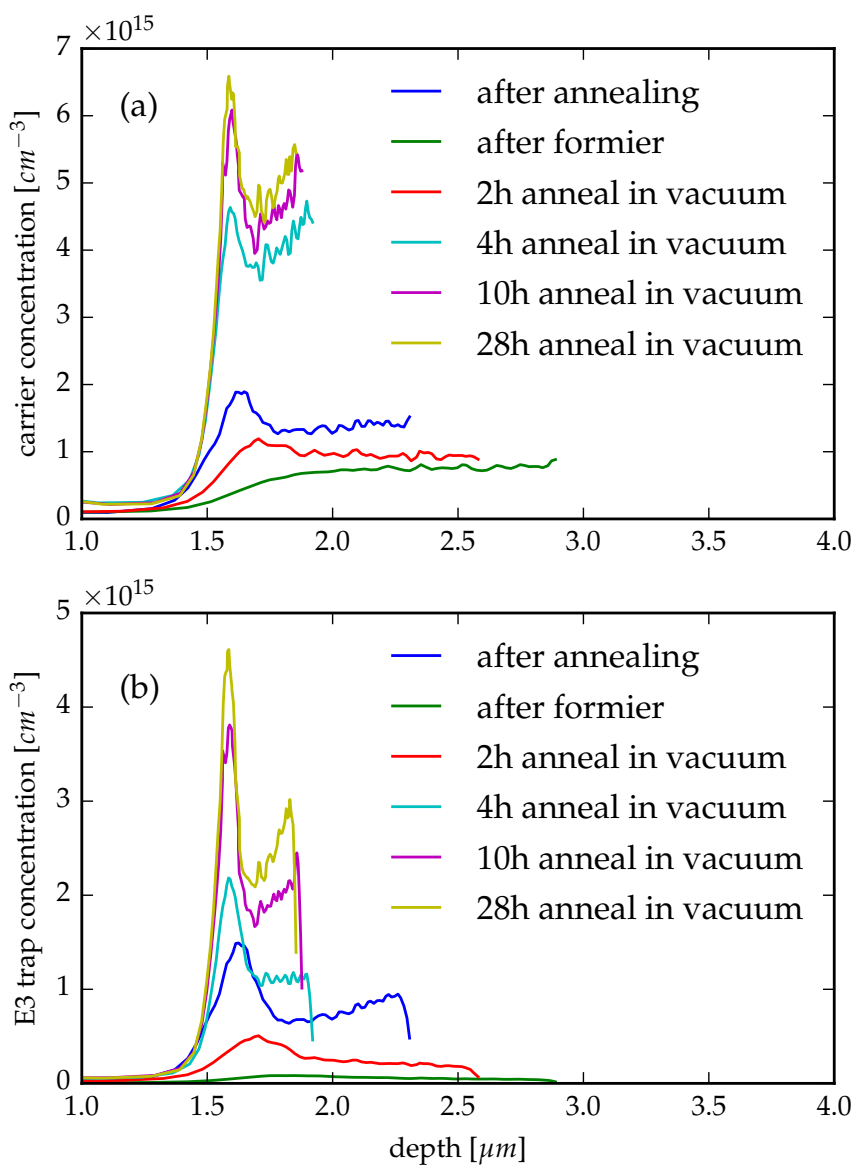

Figure 6: Dependence of the charge carrier concentration a) and the E3 trap concentration b) on exposure to a forming-gas atmosphere and subsequent isothermal annealing in vacuum at $300 \mathrm{~K}\left(<1 \times 10^{-7} \mathrm{mBar}\right)$.

after annealing in vacuum at RT for several months. Accordingly, the prevailing configurations of the passivated E3 centers are different in the two cases where the one after the forming gas treatment is presumably of higher order, involving more $\mathrm{H}$-atoms. During annealing, these high-order centers break up via a process where E3 is restored and the stable passivated configuration of E3, prevailing in the post-implant annealed samples, does not occur. That is, the release of $\mathrm{H}$ by high-order centers is not necessarily sequential with respect to $\mathrm{H}_{i}$ but could also involve $\mathrm{H}_{2}$ molecules. In the following section IV, evidence for an assignment of E3 to a $V_{\mathrm{Zn}}$ accommodating three $\mathrm{H}$-atoms, $\mathrm{H}_{3} V_{\mathrm{Zn}}$, will be put forward.

\section{THE ORIGIN OF E3}

$\mathrm{H}^{+}$and $\mathrm{D}^{+}$ions with energies in the keV range exhibit a low density of elastic energy deposition and for doses in a dilute regime, such as those employed in the present study, elementary point defects in $\mathrm{ZnO}$ prevail: $\mathrm{Zn}_{i}, V_{\mathrm{Zn}}, \mathrm{O}_{i}$ and $V_{\mathrm{O}}$. In addition, dynamic annealing 
during ion bombardment is pronounced in $\mathrm{ZnO}$, further reducing the rate of defect generation [17]. In the studied samples, $E_{F}$ is located $\sim 0.2 \mathrm{eV}$ below $E_{C}$ at RT and $V_{\mathrm{Zn}}$ appears in its double negative charge state $\left(V_{\mathrm{Zn}}^{2-}\right)$ whilst $\mathrm{Zn}_{i}$ is double positively charged acting as a shallow donor $\left(\mathrm{Zn}_{i}^{2+}\right)$ [20, 21]. $V_{\mathrm{O}}$ is a deep double donor anticipated to be in the neutral charge state 20, 21] while its $\mathrm{O}_{i}$ partner can be both electrically inactive as well as acceptor-like depending on the atomic configuration 21. It a split configuration, $\mathrm{O}_{i}$ (split), it exhibits no electrical activity but in an octahedral one, $\mathrm{O}_{i}$ (oct), it acts as a deep double acceptor. For $E_{F} \simeq\left(E_{c}-0.2\right) \mathrm{eV}, \mathrm{O}_{i}$ (oct) is thermodynamically favorable according to total energy calculations based on DFT 21 and a transformation from $\mathrm{O}_{i}$ (split) to $\mathrm{O}_{i}$ (oct) can be expected, albeit with a substantial energy barrier on the order of $\sim 1.2 \mathrm{eV}[16$. The almost one-to-one proportionality $(0.95: 1)$ between the increase in $N_{d}$ and the implanted $\mathrm{H}$ peak concentration, as found in section III A, implies a minor (if any) net charge compensation by the total of $\mathrm{Zn}_{i}, V_{\mathrm{Zn}}, \mathrm{O}_{i}$ and $V_{\mathrm{O}}$. That is, a close charge carrier balance holds with $\mathrm{O}_{i}$ (split) as the prevailing configuration and $\mathrm{H}_{i}$ as the dominant shallow donor. For the D-implanted samples, the corresponding proportionality constant is $\sim 0.3$, presumably indicating a larger concentration of $\mathrm{O}_{i}$ (oct) in this case as also expected due to the higher defect generation rate per ion.

The most obvious intrinsic defect interacting with migrating $\mathrm{H}_{i}$ 's is $V_{\mathrm{Zn}}$ because of the Coulomb attraction and the large gain of $\sim 4 \mathrm{eV}$ in total energy by the reaction $\mathrm{H}_{\mathrm{i}}{ }^{+}+V_{\mathrm{Zn}}^{2-} \longrightarrow \mathrm{H} V_{\mathrm{Zn}}^{-}[22]$. The same holds for the reaction $\mathrm{H}_{\mathrm{i}}^{+}+\mathrm{H} V_{\mathrm{Zn}}^{-} \longrightarrow \mathrm{H}_{2} V_{\mathrm{Zn}}^{0}$ leading to a fully passivated complex with zero effective charge and an energy gain of almost $3 \mathrm{eV}$ 22. The existence of $\mathrm{H} V_{\mathrm{Zn}}$ and $\mathrm{H}_{2} V_{\mathrm{Zn}}$ has been confirmed experimentally by Fourier Transform Infrared (FTIR) spectroscopy monitoring different local vibrational modes of $\mathrm{H}$ [23, 24. Further, recently Herklotz et al. 25 presented experimental evidence also for a $\mathrm{H}_{3} V_{\mathrm{Zn}}$ complex and the thermodynamic stability of such a complex, having donor-like character, was corroborated by first-principles calculations. In fact, theoretical predictions by Hupfer et al. 22 suggest that $V_{\mathrm{Zn}}$ can accommodate up to $5 \mathrm{H}$ atoms in thermodynamically stable configurations. The stability of the large $\mathrm{H}_{5} V_{\mathrm{Zn}}$ complex is promoted by self-passivation of the H's, which removes one electron from the conduction band and radically lowers the formation energy of the complex. On the basis of these considerations, the following reaction scheme is put forward for interpretation of our experimental annealing kinetics data for the E3 center:

$$
\begin{aligned}
& \mathrm{H}_{i}+V_{\mathrm{Zn}} \longrightarrow \mathrm{H}_{\mathrm{Zn}} \\
& \mathrm{H}_{i}+\mathrm{H} V_{\mathrm{Zn}} \longrightarrow \mathrm{H}_{2} V_{\mathrm{Zn}} \\
& \mathrm{H}_{i}+\mathrm{H}_{2} V_{\mathrm{Zn}} \longrightarrow \mathrm{H}_{3} V_{\mathrm{Zn}} \\
& \mathrm{H}_{i}+\mathrm{H}_{3} V_{\mathrm{Zn}} \longrightarrow \mathrm{H}_{4} V_{\mathrm{Zn}} \\
& \mathrm{H}_{i}+\mathrm{H}_{4} V_{\mathrm{Zn}} \longrightarrow \mathrm{H}_{5} V_{\mathrm{Zn}}
\end{aligned}
$$

where $\mathrm{H}_{i}$ is the only mobile species. The correspond-

\begin{tabular}{lrr}
\hline \hline & Hydrogen & Deuterium \\
{$\left[H_{i}\right],\left[D_{i}\right]$} & $2.01 \times 10^{16} \mathrm{~cm}^{-3}$ & $1.11 \times 10^{16} \mathrm{~cm}^{-3}$ \\
$\left.\left(\left[\mathrm{H}_{i}\right] /\left[V_{\mathrm{Zn}}\right]\right),\left[\mathrm{D}_{i}\right] /\left[V_{\mathrm{Zn}}\right]\right)_{t=0}$ & 4.5 & 4.6 \\
$R_{1}$ & $50 \AA$ & $48 \AA$ \\
$R_{2}$ & $40 \AA$ & $37 \AA$ \\
$R_{3}$ & $7 \AA$ & $7 \AA$ \\
$R_{4}$ & $0.15 \AA$ & $0.06 \AA$ \\
$R_{5}$ & $0.2 \AA$ & $4.1 \AA$ \\
$D_{\mathrm{H}_{i 0}}$ & $3.8 \times 10^{-3} \mathrm{~cm}^{2} \mathrm{~s}^{-1} 2.4 \times 10^{-3} \mathrm{~cm}^{2} \mathrm{~s}^{-1}$ \\
\hline \hline
\end{tabular}

Table I: Parameters used for the simulation results shown in Fig. 4 as obtained by least-squares fitting of $\left[\mathrm{H}_{3} V_{\mathrm{Zn}}\right]$ to the E3 data. $R 1$ to $R 5$ are the capture radii for reactions (1) to (5), respectively.

ing coupled differential rate equations are given in Table I] derived by applying the theory for diffusion-limited reactions [18, 19. In a first approximation, only the peak region of the implanted samples is considered and concentration gradients are neglected due to the short diffusion lengths involved with no observable profile broadening. Moreover, the influence of $V_{\mathrm{O}}$ as a competing trap for the migrating $H_{i}$ 's is omitted since the so-called E4 level, commonly associated with $V_{\mathrm{O}}$ [10, 26, 27, exhibits a generation rate of at least one order of magnitude below that of E3 in $\mathrm{H}$ implanted samples [27.

The E3 center is tentatively assigned to the $\mathrm{H}_{3} V_{\mathrm{Zn}}$ complex, both being single donors. The $\mathrm{H}_{4} V_{\mathrm{Zn}}$ complex is a double donor while $\mathrm{H}_{5} V_{\mathrm{Zn}}$ becomes a single donor because of the $\mathrm{H}$ self-passivation. As illustrated in Fig. 4, the simulated evolution of $\mathrm{H}_{3} V_{\mathrm{Zn}}$ at $300 \mathrm{~K}$ displays a close agreement with the experimental data for E3 in both the $\mathrm{H}$ and $\mathrm{D}$ samples, supporting the proposed assignment. The parameters used in the simulations are given in Table 1 and their values were obtained by least-squares fitting of $\mathrm{H}_{3} V_{\mathrm{Zn}}$ to the $\mathrm{E} 3$ data. The large capture radii of the reactions (1) and (2) reflect the Coulomb attraction between $\mathrm{H}_{i}^{+}$and the negatively charged $V_{\mathrm{Zn}}^{2-}$ and $\mathrm{H} V_{\mathrm{Zn}}^{-}$ centers. For $\mathrm{H}_{2} V_{\mathrm{Zn}}$, the radius becomes $\sim 7 \AA$ which is a typical value for an interaction determined by defect geometry and no Coulomb force present. In contrast, $\mathrm{H}_{3} V_{\mathrm{Zn}}$ exhibits a radius of only $\sim 0.15 \AA$ and $\sim 0.06 \AA$ in the $\mathrm{H}$ and $\mathrm{D}$ samples, respectively, which implies a reaction barrier on the order of $\sim 0.1 \mathrm{eV}$ of reaction (4) with $\mathrm{H}_{3} V_{\mathrm{Zn}}$ being in its neutral charge state. For $\mathrm{H}_{4} V_{\mathrm{Zn}}$, the radius is again given by defect geometry indicating that the complex is neutral at $300 \mathrm{~K}$ and thus being a deep donor. In accordance with Refs. [15, 28, the activation energy for migration of $\mathrm{H}_{i}$ and $\mathrm{D}_{i}$ was put equal to $0.6 \mathrm{eV}$ in the simulations whilst the pre-exponential factors were treated as fitting parameters. The values obtained for the pre-factors are in the range anticipated for an 'ordinary' interstitial diffusion mechanism [29], and furthermore, the ratio between the values for $D_{\mathrm{H}_{i}}$ and $D_{\mathrm{D}_{i}}$ is close to the inverse square root of their atomic mass ratio $(\sim 1.57$ versus 1.41$)$. The higher diffusivity of $\mathrm{H}_{i}$ together with its larger apparent probability for cap- 
ture in reaction (4) account for the more rapid annealing of E3 in the $\mathrm{H}$ samples than in the D ones, Fig.4. Here, it should be noted that the difference in apparent capture radius between $\mathrm{H}_{i}$ and $\mathrm{D}_{i}$ for the reaction (4) translates into a difference in energy barrier by only $\sim 25 \mathrm{meV}$ ( $\sim 90 \mathrm{meV}$ versus $\sim 115 \mathrm{meV}$ ).

The simulations yield that $\sim 65 \%$ and $\sim 30 \%$ of the implanted $\mathrm{H}$ and $\mathrm{D}$ peak concentrations are involved in the reactions (1) - (5), respectively. This is similar to the experimental results for $N_{d}$ showing a higher net concentration of $\mathrm{H}_{i}$ than of $\mathrm{D}_{i}$. Further, a ratio of $\sim 4.5$ is obtained for both $\left[\mathrm{H}_{i}\right] /\left[V_{\mathrm{Zn}}\right]$ and $\left[\mathrm{D}_{i}\right] /\left[V_{\mathrm{Zn}}\right]$ at $t=0$, which is consistent with the previous estimate for $\mathrm{D}$ ions in section III B assuming a defect survival fraction of $2 \%$ during dynamic annealing, but considerably smaller than the estimate for $\mathrm{H}$ ions. However, the spatial distributions of implanted ions and generated $V_{\mathrm{Zn}}$ 's are more confined and more separated with less straggling and overlap in the $\mathrm{H}$ samples than in the $\mathrm{D}$ ones, as revealed by SRIM simulations 12 . This reduces the probability and rate for the reactions (1) - (5) to occur in the $\mathrm{H}$ samples, i.e., the effective ratio $\left[\mathrm{H}_{i}\right] /\left[V_{\mathrm{Zn}}\right]$ becomes less than that deduced from the peak concentrations of their projected depth distributions. In addition, the overlap is also suppressed by the limited diffusion lengths involved, evidenced by no detectable broadening of the implantation profiles measured by SIMS.

To summarize, the reactions (1) - (5) with the E3 center assigned to $\mathrm{H}_{3} V_{\mathrm{Zn}}$ account reasonably well for the experimentally observed behavior of E3. This holds not only for the annealing kinetics in both the $\mathrm{H}$ - and Dimplanted samples, qualitatively also for the response of E3 on forming gas annealing, Fig.6. In the latter case, $\mathrm{H}_{3} V_{\mathrm{Zn}}$ is eventually transformed into $\mathrm{H}_{5} V_{\mathrm{Zn}}$ because of the H-rich ambient condition and where $\mathrm{H}_{5} V_{\mathrm{Zn}}$ is a deep donor being electrically neutral at RT. The assignment conforms also to results from first-principles DFT calculations of the $\mathrm{H}_{n} V_{\mathrm{Zn}}$ complexes 22. However, despite theses strong indications further work still remains for an unambiguous identification of the E3 center, e.g., DLTS measurements in combination with uniaxial sample stress should be pursued to reveal the symmetry of the center.

\section{CONCLUSION}

Using an on-line setup for in-situ DLTS and CV measurements, it is shown that the net carrier concentration $N_{d}$ in n-type $\mathrm{HT} \mathrm{ZnO}$ samples increases and saturates immediately after low dose $1 \times 10^{10}-1 \times 10^{12} \mathrm{~cm}^{-2}$ implantation by $325 \mathrm{keV} \mathrm{H}^{+}$and $\mathrm{D}^{+}$ions at $158 \mathrm{~K}$. This evidences the presence of $\mathrm{H}_{i}$ and $\mathrm{D}_{i}$, acting as shallow donors, and especially, in the H-samples an almost oneto-one proportionality holds between the implanted ion concentration and the increase in $N_{d}$. For the D-samples, the corresponding proportionality factor is lower, $\sim 0.3$, which is attributed to the higher defect generation rate per ion, enhancing the probability to form other defects involving $\mathrm{D}$ and/or compensating intrinsic defects. In contrast to $N_{d}$, [E3] does not grow immediately after the $158 \mathrm{~K}$ implant and the formation invokes a thermally activated process, most likely migration of $H_{i}\left(D_{i}\right)$. Further, the increase of [E3] is linear with ion dose, exhibiting no indication of saturation in the $1 \times 10^{15} \mathrm{~cm}^{-3}$ range. It is argued that a primary and abundant ion-induced defect is involved in the trapping of migrating $\mathrm{H}_{i}\left(\mathrm{D}_{i}\right)$ to form E3, with $V_{\mathrm{Zn}}$ as the prevailing candidate. Annealing of the implantation-induced E3 centers obeys first-order kinetics with an activation energy of $\sim 0.85 \mathrm{eV}$ and takes place already at $300 \mathrm{~K}$ on the time scale of $10-100 \mathrm{~h}$. In addition, treatment in forming gas ambient at $300 \mathrm{~K}$ gives rise to a rapid decrease of [E3]. However, E3 can be fully recovered during subsequent treatment in vacuum ambient. A kinetics model where E3 is assigned to a $\mathrm{H}_{3} V_{\mathrm{Zn}}$ complex, inferred from recent first-principles calculation results [19], and being passivated/transformed to higher order $\mathrm{H}_{n} V_{\mathrm{Zn}}$ complexes $(n>3)$ through reaction with migrating $\mathrm{H}_{i}\left(\mathrm{D}_{i}\right)$ is shown to give good quantitative agreement with the experimental annealing data.

This work was supported by the Norwegian Research Council through the FriPro program (WEDD project) and Norwegian PhD Network on Nanotechnology for Microsystems.
[1] C. G. Van de Walle and J. Neugebauer, Nature 423, 626 (2003).

[2] J. C. Simpson and J. F. Cordaro, J. Appl. Phys. 63, 1781 (1988)

[3] F. D. Auret, S. A. Goodman, M. Hayes, M. J. Legodi, H. A. van Laarhoven, and D. C. Look, Appl. Phys. Lett. 79, 3074 (2001).

[4] F. D. Auret, S. A. Goodman, M. J. Legodi, W. E. Meyer, and D. C. Look, Appl. Phys. Lett. 80, 1340 (2002).

[5] L. Vines, J. Wong-Leung, C. Jagadish, E. V. Monakhov, and B. G. Svensson, Physica B: Physics of Condensed Matter 407, 1481 (2012).

[6] L. Vines, J. Wong-Leung, C. Jagadish, V. Quemener, E. V. Monakhov, and B. G. Svensson, Appl. Phys. Lett.
100, 212106 (2012).

[7] L. Vines, E. V. Monakhov, R. Schifano, W. Mtangi, F. D. Auret, and B. G. Svensson, J. Appl. Phys. 107, 103707 (2010).

[8] V. Quemener, L. Vines, E. V. Monakhov, and B. G. Svensson, Appl. Phys. Lett. 102, 232102 (2013).

[9] W. Mtangi, M. Schmidt, F. D. Auret, W. E. Meyer, P. J. Janse van Rensburg, M. Diale, J. M. Nel, A. G. M. Das, F. C. C. Ling, and A. Chawanda, J. Appl. Phys. 113, 124502 (2013).

[10] V. Quemener, L. Vines, E. V. Monakhov, and B. G. Svensson, Appl. Phys. Lett. 100, 112108 (2012).

[11] A. Hupfer, C. Bhoodoo, L. Vines, and B. G. Svensson, Appl. Phys. Lett. 104, 092111 (2014). 
[12] J. F. Ziegler, M. D. Ziegler, and J. P. Biersack, 268, 1818 (2010).

[13] B. G. Svensson, K. H. Rydén, and B. M. S. Lewerentz, J. Appl. Phys. 66, 1699 (1989).

[14] C. G. Van de Walle, Phys. Rev. Lett. 85, 1012 (2000).

[15] K. M. Johansen, J. S. Christensen, E. V. Monakhov, A. Y. Kuznetsov, and B. G. Svensson, Appl. Phys. Lett. 93, 152109 (2008).

[16] C. Bhoodoo, A. Hupfer, L. Vines, and B. G. Svensson, to be published (2016).

[17] B. G. Svensson, C. Jagadish, A. Hallén, and J. Lalita, Phys. Rev. B 55, 10498 (1997).

[18] T. R. Waite, J. Chem. Phys. 28, 103 (1958).

[19] T. R. Waite, Phys. Rev. B 107, 463 (1957).

[20] F. Oba, A. Togo, and I. Tanaka, Phys. Rev. B 77, 245202 (2008).

[21] A. Janotti and C. G. Van de Walle, Phys. Rev. B 76, 165202 (2007).

[22] A. Hupfer, B. G. Svensson, and C. Persson, to be published (2016).

[23] E. V. Lavrov, F. Borrnert, and J. Weber, Phys. Rev. B
71, $035205(2005)$

[24] D. Bastin, E. V. Lavrov, and J. Weber, Phys. Rev. B 83, 195210 (2011).

[25] F. Herklotz, A. Hupfer, K. M. Johansen, B. G. Svensson, S. G. Koch, and E. V. Lavrov, Phys. Rev. B 92, 155203 (2015).

[26] T. Frank, G. Pensl, R. Tena-Zaera, J. Zúñiga-Pérez, C. Martínez-Tomás, V. Muñoz-Sanjosé, T. Ohshima, H. Itoh, D. Hofmann, D. Pfisterer, et al., Appl. Phys. A 88, 141 (2007).

[27] A. Hupfer, C. Bhoodoo, L. Vines, and B. G. Svensson, J. Appl. Phys. 119, 181506 (2016).

[28] M. Wardle, J. Goss, and P. Briddon, Phys. Rev. Lett. 96, 205504 (2006)

[29] P. M. Fahey, P. B. Griffin, and J. D. Plummer, Rev. Mod. Phys. 61, 289 (1989).

[30] D-samples were used for the SIMS measurements rather than H-samples because of an improved detection limit by about two orders of magnitude $\left(10^{15}\right.$ versus $\left.\left.10^{17} \mathrm{~cm}^{-3}\right)\right)$. 